\title{
Referencia al artículo "Uso de la urografía por resonancia magnética en el estudio del aparato urinario frente a la urografia convencional"
}

\author{
Sánchez-Martín FM. \\ Servicio de Urología. Fundación Puigvert. Barcelona
}

Actas Urol Esp. 2007;31(5):567-568

\section{Sr. Director:}

En referencia al trabajo de Ramos de Campos et al. titulado "Uso de la urografía por resonancia magnética en el estudio del aparato urinario frente a la urografia convencional", lo autores incluyen entre las indicaciones para la realización de uroRM (o urografia por resonancia magnética) la insuficiencia renal (IR), con 27 casos de fracaso renal agudo. La uroRM suele implicar el empleo de gadolinio, un medio de contraste paramagnético no iónico que se elimina por filtración glomerular, por lo que.proporciona un realce de la vía urinaria durante la obtención de imágenes por RM.

En febrero de 2007, la Agencia Española de Medicamentos y Productos Sanitarios (AEMPS) emitió una nota informativa sobre novedades en cuanto a la seguridad en la administración del gadolinio (en especial la gadodiamida, Omniscan® ${ }^{1}{ }^{1}$ para la realización de $\mathrm{RM}$, asociándola a la aparición de Fibrosis Sistémica Nefrogénica (FSN) ${ }^{2}$, también llamada Dermopatía Fibrosante Nefrogénica $(\mathrm{DFN})^{3}$.

La FSN se caracteriza por la formación de tejido conectivo en la piel, que aparece engrosada, áspera y dura, pudiendo producir contracturas con disminución de la movilidad de las articulaciones. Puede afectar también músculos, serosas, corazón y pulmón ${ }^{4}$. La FSN se presenta en pacientes con IRC grave y en trasplantados hepáticos con IR1, no estando descrita en pacientes con función renal normal ${ }^{2}$.

La AEMPS y la Agencia Europea de Medicamentos (EMEA) ${ }^{6}$ avisan que los contrastes para $\mathrm{RM}$ que contienen gadodiamida están contraindicados en pacientes con IR grave o con transplante hepático. Durante el embarazo y hasta el primer año de vida sólo deben administrase en casos cuidadosamente evaluados, debido a la inmadurez de la función renal. Respecto al resto de quelatos de gadolinio sólo pueden administrarse a pacientes con IR grave tras una profunda valoración del balance riesgo-beneficio individual.

Los autores señalan la importancia de valorar la función renal para limitar la nefrotoxicidad del gadolinio, en especial cuando la creatinina supera los $2 \mathrm{mg} / \mathrm{dL}$, pero sin remarcar el riesgo de FSN en pacientes con IR. Creemos relevante observar que la indicación clásica de uroRM en situación de IR ha cambiado recientemente, quedando contraindicado el empleo de gadolinio en pacientes con tasa de filtración glomerular-TFG < $30 \mathrm{ml} / \mathrm{min} / 1,73 \mathrm{~m} 2$. Asimismo la administración durante el embarazo ha de ser fruto de una cuidadosa valoración riesgo-beneficio. La presente información puede complementar los datos aportados por los autores de este interesante trabajo sobre utilidad de la uroRM.

Fdo.: F.M. Sánchez-Martin

\section{REFERENCIAS}

1. Ficha técnica y Prospecto. Omniscan®.

2. Fibrosis sistémica nefrogénica y contrastes de Gadolinio para resonancia magnética. Agencia española de medicamentos.

3. Boyd AS, Zic JA, Abraham JL. Gadolinium deposition in nephrogenic fibrosing dermopathy. J. Am. Acad. Dermatol. 2007 Jan;56(1):27-30.

4. Stöllberger C, Finsterer J. Dermal and extra-dermal manifestations of gadolinium-triggered nephrogenic fibrosing dermopathy. Nephrol. Dial. Transplant. 2006 Dec;21(12): 3604; author reply 3604-5.

5. Baron PW, Cantos K, Hillebrand DJ, Hu KQ, Ojogho ON, Nehlsen-Cannarella S, Concepcion W. Nephrogenic fibrosing dermopathy after liver transplantation successfully treated with plasmapheresis. Am J Dermatopathol. 2003 Jun;25(3):204-9

6. European Medicines Agency Post-Authorisation Evaluation of Medicines for Human Use. Vasovist and nephrogenic systemic fibrosis (NSF). 


\section{RÉPLICA A LA CARTA AL DIRECTOR}

Como réplica a la carta remitida por parte del Dr. Sánchez-Martín en relación al trabajo publicado en esta revista el pasado mes de marzo, debo reseñar que, si bien, la Fibrosis Sistémica Nefrogénica (FSN) es una grave entidad nosológica que podría estar asociada al empleo del gadolinio, realizar dicha puntualización en relación a nuestra publicación podría complementar la información en relación al uso de contrastes paramagnéticos del tipo gadolinio, función que ya realiza la Agencia Española de Medicamentos y Productos Sanitarios ${ }^{1}$, aunque no considero preciso vincular a este estudio. Principalmente, debo apuntar que la urografía por resonancia magnética o urorresonancia (UroRM) es una modalidad que obtiene las imágenes urográficas gracias a secuencias altamente potenciadas en $\mathrm{T} 2 \mathrm{w}$, es decir, secuencias que realzan las señales procedentes del fluido estático corporal (fluido cerebroespinal, humor acuoso, intestinal, hepatobiliopancreático, quistes y sistema excretor urinario), gracias al software empleado, sin precisar en ningún momento el uso de contrastes paramagnéticos. Los estudios con contraste como el gadolinio permiten ocasionalmente complementar la exploración. Generalmente, se emplean en aquellos casos donde es preciso una valoración funcional del parénquima renal, para realizar el diagnóstico diferencial entre un tumor de vías captante de contraste frente a otras lesiones como coágulos o litiasis en la vía, así como para mejorar la calidad de la imagen urográfica siempre que el paciente no presente en asociación una afectación severa de la función renal, tal y como se explica en el algoritmo de la ejecución de técnica en la Tabla 3. En el trabajo han sido incluidos 27 pacientes con fracaso renal agudo o crónico, pero como se explica en la discusión de la publicación y siguiendo el algoritmo de la técnica, ningún caso con afectación severa fue expuesto al gadopentato de dimeglumina (gadolinio empleado en nuestro centro), es más, la precaución en la práctica clínica restringe más su uso que en los supuestos teóricos ${ }^{2}$.

$\mathrm{El}$ conocimiento en materia de resonancia magnética de la FSN es preciso aunque no debe suponer un motivo de alarmismo hacia esta exploración. Esta grave entidad nosológica fue descrita por vez primera en Marzo de $1997^{3}$, y aunque si bien su conocimiento no es reciente, sigue sin estar clara su etiopatogenia. Desde enero de 2000 hasta Octubre de 2006 se han administrados 40 millones de dosis de gadolinio en Estados Unidos, apareciendo 215 casos de FSN (3 fallecimientos) ${ }^{4}$. La incidencia en Europa es similar, sin embargo, todos los casos europeos se han asociado a la gadodiamida, mientras que en Estados Unidos la mayoría de casos se expusieron a gadodiamida pero un pequeño porcentaje se ha relacionado con la gadoversetamida y el gadopentato de dimeglumina. Parece ser que la formulación de la gadodiamida difiere notablemente en relación a los otros contrastes, presentando un exceso de quelato (12 $\mathrm{mg} / \mathrm{ml})$ que lo hace menos estable. Su asociación con la insuficiencia renal severa podría explicarse por el depósito tisular extracelular de esta molécula al no trasportarse vinculada a proteínas, es decir, por exceso de fracción libre. De todas formas, el hecho de que se haya registrado casos de FSN sin exposición al gadolinio, así como de que no se desarrolle necesariamente la enfermedad tras su exposición en contexto de insuficiencia renal severa, apunta a que el gadolinio actúe muy probablemente como cofactor, siendo preciso que incurran otras circunstancias que actualmente se desconocen ${ }^{5}$.

Resulta fundamental para el empleo óptimo de las herramientas que disponemos conocer todos los posibles factores asociados, siendo esencial en materia de contrastes paramagnéticos el conocimiento de la FSN, por lo que agradecemos al Dr. Sánchez- Martín el comentario realizado al respecto, destacando que el trabajo que hemos publicado respeta el uso del gadolinio según las indicaciones de la AEMPS, sin exponer innecesariamente a esta molécula si el beneficio no es suficiente, y más en nuestro caso, donde la obtención de las secuencias urográficas mayoritariamente no precisa de contraste alguno.

\section{REFERENCIAS}

1. http://www.agemed.es/actividad/alertas/usoHumano/ seguridad/2007_03_NI_GADOLINIO_Y_FSN.htm.

2. Garcia-Valtuille R, Garcia-Valtuille AI, Abascal F, Cerezal L, Arguello MC. Magnetic resonance urography: a pictorial overview. Br J Radiol. 2006;79(943):614-626.

3. Aydingoz U. The need for radiologists' awareness of nephrogenic systemic fibrosis. Diagn Interv Radiol. 2006;12(4):161-162.

4. Cowper SE, Su LD, Bhawan J, Robin HS, LeBoit PE. Nephrogenic fibrosing dermopathy. Am J Dermatopathol. 2001;23(5):383-393.

5. Thomsen HS. Nephrogenic systemic fibrosis: A serious late adverse reaction to gadodiamide. Eur Radiol. 2006; 16(12):26192621.

Fdo.: M. Ramos de Campos. Servicio de Urología. Consorcio Hospital General de Valencia. 